\title{
Cobertura de Citología Vaginal en Mujeres mayores de 15 años
}

\author{
SABANETA - ANTIOQUIA \\ 1986
}

Dres.: Luz Magdalena Zuluaga P.*, Doralba Jaramillo de J.**

\section{RESUMEN}

Con el fin de conocer la cobertura del programa de citología cervico-vaginal y el conocimiento que tiene la población femenina en mayores de 15 años sobre el tema, se realizó un estudio en una muestra de 608 mujeres mayores de 15 años representantes de las mujeres adultas del Municipio de Sabaneta. La información se obtuvo a través de entrevistas realizadas en el domicilio de las mujeres seleccionadas en la muestra. La cobertura del programa regular de citología cervico-vaginal fue de $32.56 \times 100$ mujeres adultas.

De las variables demográficas y gineco-obstétricas analizadas como factores que podían influir en la realización regular de la citología fueron positivas: Edad de 25 a 49 años y el antecedente de embarazo y actividad sexual. El 66\% de las razones expuestas para no realizar regularmente la citología, son causas que se pueden abolir con ampliación de los servicios de salud y mayor educación; puesto que el $32.1 \%$ de las mujeres que alguna vez se practicaron citología vaginal no recibieron ninguna instrucción sobre el tema al momento del procedimiento. Al analizar la última citología se observó que el $19.3 \%$ fueron positivas (grado III-IV o inflamatorios) de éstas el $48.8 \%$ fueron realizadas en Instituciones privadas; del total de las personas que poseen seguridad social sólo el $40.9 \%$ utilizó dichos servicios. Al evaluar la institución sobre el tema se encontró deficiencia de conocimeintos en los resi-

* Profesora Métodos Investigación Epidemiológica, Facultad de Enfermería, Universidad de Antioquia.

** Profesora Principios de Enfermería, Facultad de Enfermería Universidad de Antioquia. dentes del área rural, en el nivel económico alto y bajo, en las edades de $15-24$ y 50 y más años y entre quienes no tienen antecedentes personales de actividad sexual o embarazo.

\section{INTRODUCCION}

En Colombia la patología oncológica es un componente importante de la morbi-mortalidad, siendo la primera causa de muerte para el grupo de edad de 45 y más ã̃os.

Respecto al Cáncer de Cuello Uterino ocupa el segundo lugar de frecuencia según la localización del cáncer en mujeres por lo tanto es considerado como uno de los diez enemigos de la salud del colombiano sometida a programas especiales para su control (1).

A nivel del Departamento de Antioquia la patología oncológica figura como la segunda causa de egresos hospitalarios para 1985 (2) y la quinta causa de mortalidad general con una tasa de $26 \times 10^{-5}$, para 1986 (3).

Analizando la tendencia de mortalidad (Gráfico 1) se observa un incremento en el tiempo, posiblemente por el aumento de la expectativa de vida y de la edad promedio de la población.

El cáncer ginecológico corresponde al $60 \%$ del total de las patologías oncológicas de la mujer (4) siendo el cáncer de cuello uterino el más incidente con tasas de 44.7 × 100.000 mujeres mayores de 15 años para 1985 (5).

Experiencias en otros países han demostrado que después de introducir programas de tamizaje con citología cervico vaginal periódica se reduce la 
mortalidad por cáncer de cuello uterino en mujeres mayores de 25 años (6).

\section{GRAFICO 1}

DISTRIBUCION PORCENTUAL DE LA MORTALIDAD POR TUMORES MALIGNOS DEPARTAMENTO DE ANTIOQUIA 1943-1986

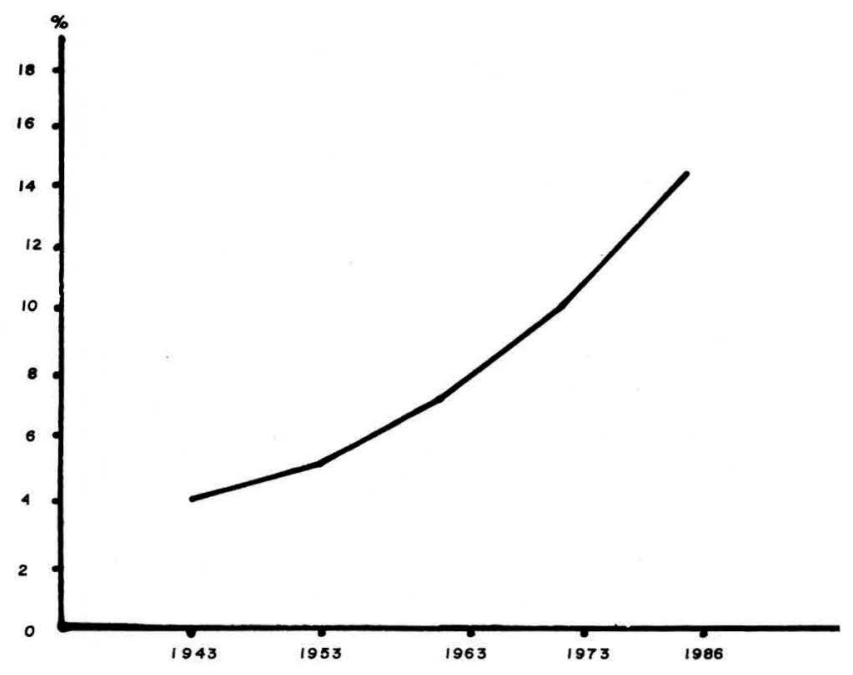

FUENTE: CERTIFICADOS DE DEFUNCION

Las experiencias de Connecticut que tiene el registro de cáncer más antiguo de U.S.A. (7) y Finlandia donde el programa de tamizaje fue introducido en 1963 (8) se observa un aumento en la incidencia del carcinoma en estadio In-situ concomitante con una disminución de la incidencia y mortalidad del cáncer invasivo (Gráfico 2 y 3 ).

\section{GRAFICO 2}

TASA DE INCIDENCIA DE GANCER IN-SITU AJUSTADO POR EDAD Y TASA DE INCIDENCIA Y MORTALIDAD DE CA INVASIVO CERVICAL POR 100.000 MUJERES EN CONNECTICUT. 1935 - 1973.

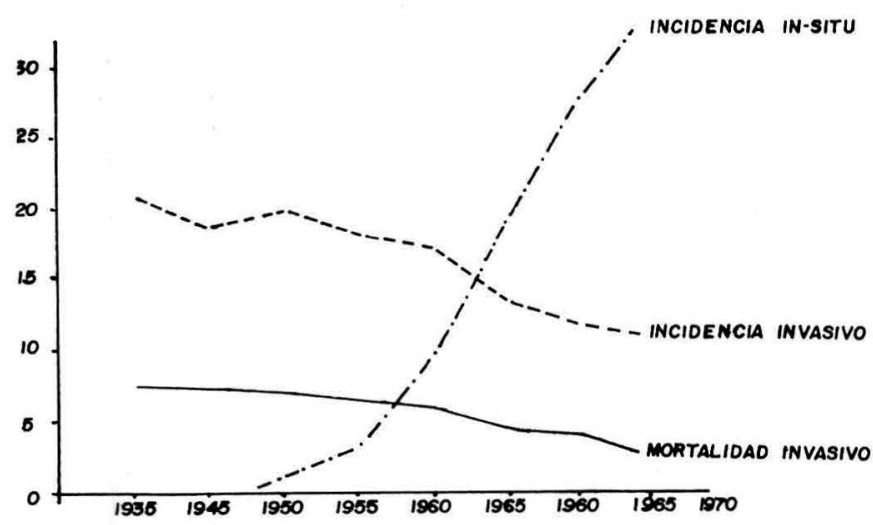

\section{GRAFICO 3}

CANCER DE GUELLO UTERINO EN FINLANDIA. TASA DE INCIDENCIA Y MORTALIDAD DE CANCER INVASIVO AJUSTADO POR EDAD Y TASA DE DETECCION DE LESIONES DE CARCINOMA IN-SITU 1953-1974 MORTALIDAD PROVISIONAL PARA 1976

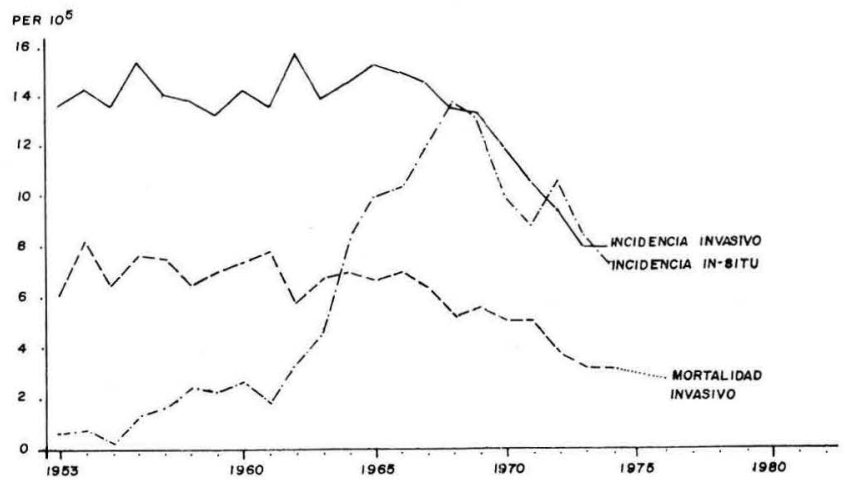

En el Departamento de Aritioquia la práctica de la citología cervico-vaginal regular se inició en el año de 1975 como componente del programa de planificación familiar.

Sin embargo, las tasas de mortalidad por cáncer de cuello uterino no han mostrado el descenso esperado en los 12 años de existencia del programa. Así es como para el período de tiempo 1982-1986 se observan tasas en el departamento de: 12.99; $12.27 ; 10.53 ; 10.66$ y 9.35 por 100.000 mujeres mayores de 15 años respectivamente (5).

En igual forma si se analiza el estadio del Cáncer al momento del diagnóstico utilizando como indicador la razón entre el diagnóstico en estadio Insitu con relación al estadio IIIb. Dx In-situ/Dx grado IIIb información obtenida del grupo oncológico del Hospital San Vicente de Paúl de los años 19801985, hospital estatal de máxima tecnología, se observan cifras por año de: $0.78 ; 1.69 ; 1.05 ; 0.99$; $1.26 ; 0.75$ respectivamente (9) lo que demuestra que el diagnóstico invasivo no se ha reducido en este período en forma significativa.

Los dos indicadores analizados; mortalidad y estadio del carcinoma al momento del diagnóstico, muestra el poco impacto que el programa ha producido en la población para el diagnóstico precoz de cáncer de cuello uterino.

Por el análisis anterior se justifica realizar un estudio que mida el impacto en la comunidad de este programa. 


\section{METODOLOGIA}

Con el objetivo de medir la cobertura del programa de detección precoz del cáncer de cuello uterino por medio de la toma regular de la citología cérvico-vaginal y los conocimientos que sobre el tema tiene la población femenina mayor de 15 años, se hizo una encuesta en una muestra representativa de la población de Sabaneta.

El Municipio está ubicado al Sur de la ciudad de Medellín, tiene una extensión de $15 \mathrm{~km}^{2}$ de los cuales 3.95 son urbanos y 11.05 son rurales. Además cuenta con una población de 21.784 habitantes. De éstos, 8.304 corresponden a mujeres mayores de 15 años. Según proyección para 31-XII-86, presenta una tasa de crecimiento poblacional de $4.68 \%$ para el área urbana y de $2.42 \%$ para el área rural.

La densidad población bruta total es de 1.452 habitantes $/ \mathrm{km}^{2}$ correspondiendo el área urbana a 4.134 habitantes $/ \mathrm{km}^{2}$ y 1.306 habitantes $/ \mathrm{km}^{2} \mathrm{pa}$ ra el área rural.

La información se obtuvo a través de entrevistas realizadas en el domicilio de la totalidad de mujeres que habitan en el sector seleccionado en la muestra y que fueron mayores de 15 años, obteniéndose información sobre variables demográficas. Lugar de residencia, nivel económico y edad.

Además variables gineco-obstétricas: Activiđad sexual, número de embarazos, datos sobre la práctica de la citología, tiempo resultado, razones de no realizarse la citología, institución que la realizó, instrucciones que recibieron las usuarias y los conocimientos que dichas mujeres tenían sobre la citología vaginal.

Para el análisis estadístico de los datos se calcularon probabilidades de práctica regular de citología y de conocimientos sobre el tema según las variables demográficas y gineco-obstétricas.

Se analizó la distribución porcentual de los distintos aspectos de la práctica de la citología como prueba de dosimacia, diferencia de proporciones y chi cuadrado.

La muestra fueron 608 mujeres mayores de 15 años, se empleó un diseño muestral proporcional por lugar de residencia (urbana y rural). Como marco muestral se utilizó la cartografía e información del censo poblacional de 1985.

\section{RESULTADOS}

Para evaluar la representatividad del estudio se comparó la composición poblacional por edad y lugar de residencia con la composición de la muestra siendo iguales estadísticamente para las mayores de 25 años y mayor la muestra para el grupo etario de 15 a 24 años. (Gráfico 4).

\section{GRAFICO 4}

COMPOSICION POR EDAD DE LA POBLACION Y MUESTRA

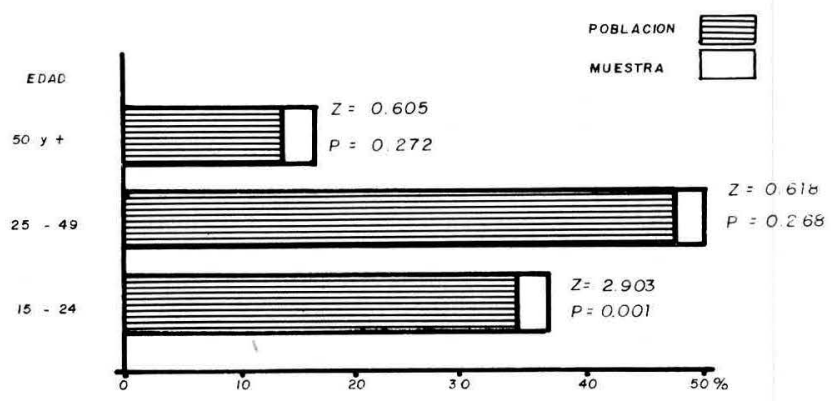

En cuanto al lugar de la residencia fueron similares estadísticamente, en el área urbana la población residente $56.4 \%$ y $54.7 \%$ de la muestra, la distribución rural fue de $43.6 \%$ de la población y $45.3 \%$ de la muestra $x^{2}=0.915 \mathrm{p}=0.16$. Dichos resultados confirman la representatividad de la muestra y por lo tanto las conclusiones del presente estudio se pueden inferir a la población de $\mathrm{Sa}$ baneta.

La cobertura del programa de toma regular de citología vaginal para el diagnóstico precoz del cáncer de cuello uterino fue del $32.6 \%$ (198) de las mujeres adultas (mayores de 15 años). Es importante destacar que al 38,7\% (235) de las mujeres encuestadas, nunca se les ha practicado una citología vaginal. (Gráfico 5).

$\mathrm{Al}$ analizar las variables demográficas que pudieron influir en la práctica periódica de la citología vaginal se encontró que: El lugar de residencia y el nivel económico no contribuyeron. En cambio, fue más frecuente la práctica de la citología vaginal en las edades de 25 a 49 años y el haber tenido embarazos. Ninguna mujer que no haya tenido vida sexual activa se realizó periódicamente la citología vaginal (Tabla 1 ).

Al analizar el tiempo transcurrido desde la última citólogía sólo el $48.8 \%$ tuvieron un tiempo menor de dos años. (Tabla 2). 
GRAFICO 5

DISTRIBUCION SEGUN LA PRACTICA DE REALIZACION CITOLOGIA VAGINAL

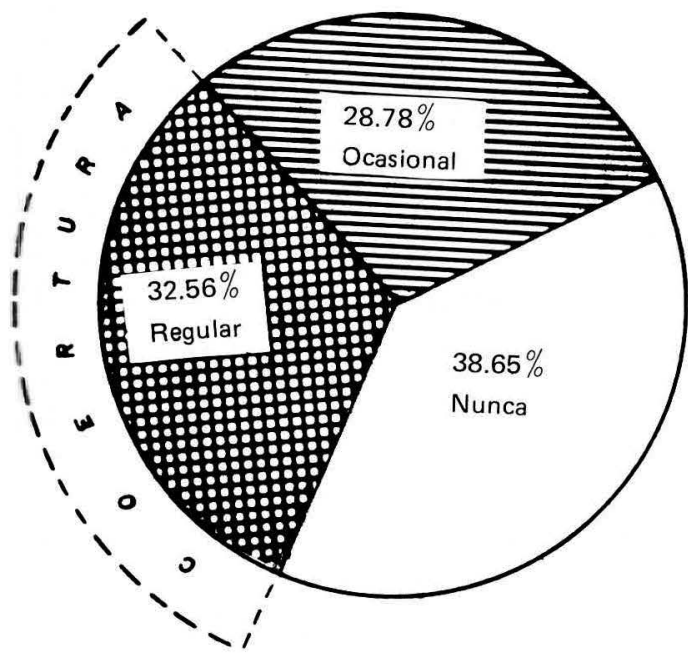

TABLA 1

PROBABILIDAD X 100 DE LA PRACTICA PERIODICA DE LA CITOLOGIA VAGINAL SEGUN VARIABLES DEMOGRA FICAS Y GINECO-OBSTETRICAS

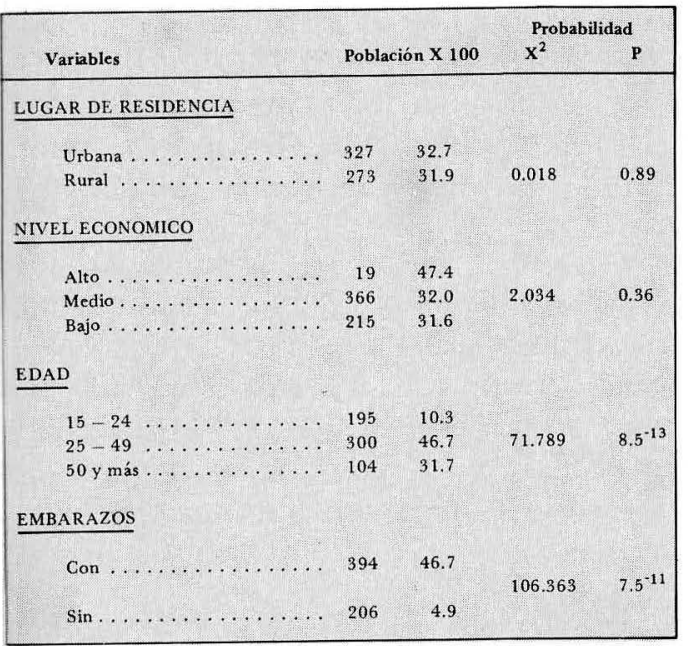

Las razones por las cuales las entrevistadas no realizaban la citología periódicamente, en el 66\% son casos no justificables, que con coberturas adecuadas de atención o educación en salud se abolirían. (Tabla 3). Se clasificó "no necesita" las mujeres de 25 años que no tienen vida sexual activa.

E1 $32.1 \%$ (120) de las mujeres que alguna vez se practicaron citología vaginal no recibió instrucción al conocer el resultado. En este momento se podría reforzar la instrucción sobre la utilidad de esta práctica para el DX Precoz del cáncer (Tabla 4).

TABLA 2

TIEMPO TRANSCURRIDO DESDE LA ULTIMA CITOLOGIA

\begin{tabular}{|l|c|c|c|}
\hline $\begin{array}{c}\text { Meses } \\
\text { Transcurridos }\end{array}$ & No. caso & Porcentaje & $\begin{array}{c}\text { Porcentaje } \\
\text { acumulado }\end{array}$ \\
\hline $1-6$ & 148 & 24.5 & 24.5 \\
$7-24$ & 147 & 24.3 & 48.8 \\
$25-36$ & 29 & 4.8 & 53.6 \\
37 y más & 45 & 7.5 & 61.1 \\
Nunca Citología & 235 & 38.9 & 100 \\
\hline
\end{tabular}

NOTA: 4 casos no informaron.

TABLA 3

\section{RAZONES POR LAS CUALES NO ACOSTUMBRAN} REGULARMENTE LA CITOLOGIA

\begin{tabular}{|l|c|c|c|}
\hline \multicolumn{1}{|c|}{ Razones } & No. casos & Porcentaje & $\begin{array}{c}\text { Porcentaje } \\
\text { acumulado }\end{array}$ \\
\hline Económicas & 12 & 3 & 3.0 \\
Culturales & 158 & 38.9 & 41.9 \\
Negligencia & 98 & 24.1 & 66.0 \\
No lo necesita & 138 & 34.0 & 100 \\
\hline
\end{tabular}

TABLA 4

\section{CONDUCTA A SEGUIR DESPUES DEL RESULTADO DE CITOLOGIA}

\begin{tabular}{|l|c|c|}
\hline \multicolumn{1}{|c|}{ Conductas } & No. casos & Porcentaje \\
\hline Control en un año o 6 meses & 194 & 52.0 \\
Tratamiento y nuevo control & & \\
3 a 6 meses & 1 & 14.5 \\
Repetición del examen & & 0.3 \\
Repetición del examen y & 4 & 1.1 \\
consulta al especialista & 120 & 32.1 \\
\hline No recibió ninguna información
\end{tabular}

De las 350 entrevistadas que informaron resultados de la última citología el $14.3 \times 1.000$ (5) fueron positivas (grado IV) y el $11.4 \times 1.000$ (4) sospechosas (grado III). 
Se obtuvo información de 363 entrevistadas sobre la institución donde se practicaron la última citología. En instituciones del estado 37.4\% (136); por alguna institución de seguridad social $13.8 \%$ (50) y por médico privado el $48.8 \%$ (177).

En $33.6 \%$ (122) informaron tener algún sistema de seguridad social. De éstas sólo el $40.9 \%$ utilizaron el servicio.

Otro de los objetivos propuestos en el trabajo fue medir el conocimiento que la población tiene sobre la citología cérvico-vaginal como método diagnóstico del cáncer de cuello uterino.

Para el logro de dicho objetivo se realizaron una serie de preguntas:

A la primera pregunta, sobre la utilidad de la citología vaginal sólo el 50.5\% (305) dieron una respuesta correcta. Cuando se analizó la calidad de la respuesta según las variables demográficas y ginecoobstétricas, tuvieron mayor conocimiento la población urbana y las de clase media económica.

E1 $4.5 \%$ (27) consideran que la citología vaginal sólo se la pueden realizar las mujeres cuando planifican. Dicho concepto es mayor en las mujeres sin actividad sexual y que no han tenido embarazos.

La creencia de que sólo las mujeres que tienen hijos se deben hacer la citología vaginal, la tienen $6.7 \%$ (40) de las mujeres entrevistadas. Esta creencia es mayor en las mujeres de 15 a 24 años y las mayores de 50 años, como también, las que no han tenido actividad sexual o embarazos.

Al interrogar sobre si las mujeres que no han tenido relaciones sexuales se pueden practicar citología, el 20.7\% (124) respondieron que no. Dicho concepto es mayor en la población rural, en edades de 15 a 24 años y mayores de 50 , que no han tenido relaciones sexuales ni hijos, (Tabla 5 ).

Entre las razones por las cuales no se debe practicar dicho examen las más frecuentes fueron:

Porque se puede perder la virginidad $=38$

Si no tienen relaciones sexuales no necesitan $=35$

Si es virgen no tiene por que sufrir esas enfermedades $=11$

Sólo casadas se la pueden hacer $=7$

Otros $=2$

No sabe explicar $=17$

Sin información $=25$
TABLA 5

\section{PORCENTAJE DE CONOCIMIENTOS SOBRE CITOLOGIA VAGINAL (V.C.) SEGUN VARIABLES DEMOGRAFICAS Y GINECO-OBSTETRICAS}

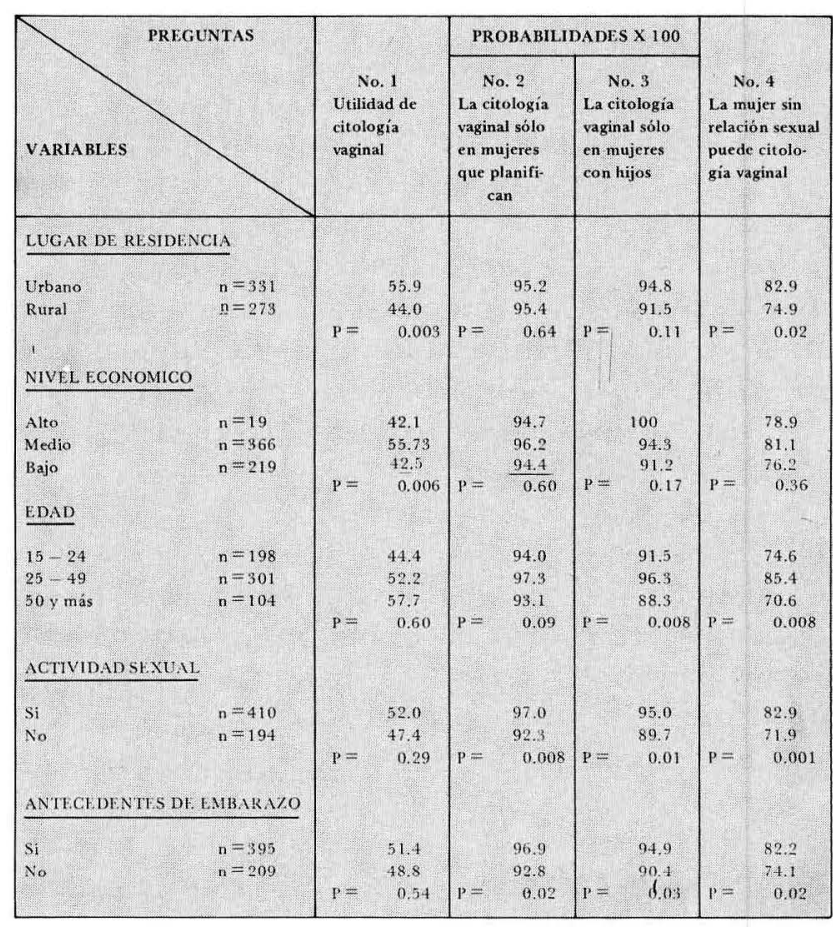

\section{DISCUSION Y CONCLUSIONES}

El poco impacto del programa de citología cérvico-vaginal en la reducción de mortalidad por cáncer de cuello uterino en el Departamento, en parte es explicable por las bajas coberturas de este programa, teniendo en cuenta que el $38.7 \%$ de las mujeres encuestadas nunca se han realizado una citología y sólo el $32.6 \%$ informan tener una práctica regular de toma de ella.

Estas cifras son bajas si se comparan con coberturas de países que han logrado reducir la mortalidad de cáncer de cuello uterino como es el caso de EE.UU. que informa que el $50 \%$ de las mujeres mayores de 17 años han realizado una citología en el año (10).

La población donde se realizó el estudio es el campo de práctica de las áreas de la salud de una universidad privada, por lo tanto las coberturas en el departamento son más bajas.

El S.S.S.A. informa para el Departamento de Antioquia en 1987 coberturas del 6.6\% (11) (cito- 
logías realizadas por instituciones del estado/población usuaria potencial femenina mayores de 15 años).

Es importante destacar el hecho de que las entrevistadas que se practicaron citología sólo el $37.4 \%$ la hicieron en instituciones del Estado.

De las mujeres que informaron tener cualquier sistema de seguridad social el $40.9 \%$ utilizan este recurso para la toma de citología vaginal, sugiere una subutilización de los servicios de salud prepagados. La cobertura de la toma de citología vaginal se ampliaría, aumentando los recursos de salud y mejorando la instrucción sobre el tema, puesto que el 66 . de las justificaciones para no realizarse la citología son reducibles.

Se puede concluir que la instrucción sobre el tema es deficiente debido a que el $32.1 \%$ de las mujeres que se realizan la citología no recibieron información de los servicios de salud al momento del procedimiento. Además al evaluar los conocimientos mostraron mayor ausencia la población rural, las edades extremas ( 15 a 24 años y mayores de 50 ) y sin vida sexual activa. Es preocupante el hecho de que en la juventud no estén claros estos conceptos cuando el estado colombiano exige en el currículo escolar información sexual.

El porcentaje de citología vaginal positiva en el estudio fue de $14.3 \times 1.000$ resultados conocidos. Es alta si se se compara con los índices de po- sitividad informados por el S.S.S.A. y 5.6 x 1.000 para el Departamento de Antioquia y el Valle de Aburrá, Regional a la que pertenece Sabaneta (11).

La actividad sexual parece ser un factor determinante para la práctica regular de la citología puesto que ninguna de las mujeres que informan no tener actividad sexual se toman regularmente la citología.

Entre las variables demográficas y gineco-obstétricas analizadas que pueden influir en la toma regular de citología se encuentran: Tener una edad entre 25 y 40 años, y tener antecedentes de práctica sexual y embarazos.

\section{AGRADECIMIENTOS}

Los autores expresan sus agradecimientos:

A Rosa Elena Correa de G., Profesora de Atención Materno Infantil, Facultad de Enfermería, Universidad de Antioquia.

A Clara Inés Rico de A., Estudiante Universidad Austín Texas.

A Ana María Zuluaga de V., Estudiante Universidad Pontificia Bolivariana.

A Isabel Cristina Alvarez V., Estudiante Universidad de Antioquia.

\section{BIBLIOGRAFIA}

1. COlOMbia, Ministerio DE SALUd. Cambio con Equidad: Potíticas y Realizaciones 1983-1984, Bogotá: El Ministerio 1984, p. 24-34.

2. ANUARIO ESTADISTICO DE ANTIOQUIA. 1985. Mede1lín, Departamento Administrativo de Planeación 1986 p. 131.

3. ANTIOQUIA SERVICIO NACIONAL DE SALUD. Situaciones de Salud. Medellín S.S.S.A. 1987 p. 17-34.

4. DUQUE, A.; CARVAJAL, P. Egresos por Cáncer en el Departamento de Antioquia 1978-1980. En Boletín Epidemiológico de Antioquia 7 (3): 84-88 julio, septiembre, 1982.

5. MICROFICHAS MORTALIDAD. Información del S.S.S.A., 1985.

6. MUNOZ, N. Epidemiología del Cáncer del Cuello Uterino; Curso de Epidemiología del Cáncer Lima, Perú. Febrero 27 marzo 9 de 1984 (mimeografiado).
7. LASKEY P.; MEIGI, J.W.; FLANNERY, J.T. Uterine Cervical Carcinoma in Connecticut 1935-1973. Evidence of two classes of invasive disease J. Natl. cancer Inst. 57: 1037-1043 1976.

8. HAKAMA, M.; MASS SCREENING. For cervical cancer in Finland. In: Screening and cancer VICC technical Report. Vol. 40, 1978.

9. ARCHIVOS del Grupo de Oncología del Hospital Universitario San Vicente de Paúl. Medellín, Colombia.

10. FOLTZ, A.M.; KELSEY, J.L. Es Realmente útil la Práctica Anual de la Prueba de Papanicolaun. Foro Mundial de la Salud $1(1,2)$ : 124-136, 1980.

11. S.S.S.A. Informe Materno Infantil, 1987. Sin publicar. 